\title{
Biologic Therapy for Psoriatic Arthritis or Moderate to Severe Plaque Psoriasis: Systematic Review with Pairwise and Network Meta-Analysis
}

\author{
Mariangela Peruzzi ${ }^{1}$, Delia Colombo ${ }^{2}$, Elena De Falco ${ }^{1}$, Isotta Chimenti ${ }^{1}$, Antonio Abbate ${ }^{3}$, \\ Giacomo Frati ${ }^{1,3}$ and Giuseppe Biondi-Zoccai ${ }^{1, *}$ \\ ${ }^{1}$ Department of Medico-Surgical Sciences and Biotechnologies, Sapienza University of Rome, Latina, Italy \\ ${ }^{2}$ Novartis, Origgio, Italy \\ ${ }^{3}$ VCU Pauley Heart Center, Virginia Commonwealth University, Richmond, VA, USA \\ ${ }^{4}$ Department of AngioCardioNeurology, IRCCS Neuromed, Pozzilli, both in Italy
}

\begin{abstract}
Background: A comprehensive assessment of the risk-benefit profile of biologic agents in psoriasis is lacking. We conducted a network meta-analysis of randomized trials on biologic agents in psoriasis.

Methods: Trials on biologic agents in psoriasis (including psoriatic arthritis) were sought in several databases. Endpoints were $\geq 75 \%$ Reduction in the Psoriasis Area and Severity Index (PASI75), $\geq 20 \%$ improvement in the American College of Rheumatology core set of outcomes (ACR20), serious adverse events (SAE), and adverse events (AE) at the longest available non-cross-over follow-up. Random-effect methods were used to obtain pairwise and network pooled estimates.

Results: A total of 52 trials with 17,617 patients and 9 different biologic agents included, with $52 \%$ affected by psoriatic arthritis. After an average follow-up of 18 weeks, treatment with placebo was associated with a $5.9 \%(5.2 \%-6.6 \%)$ rate of PASI75, 17.4\% (15.1\%-19.6\%) of ACR20, 2.4\% (1.9\%-2.8\%) of SAE, and 51.8\% (50.2\%-53.4\%) of AE. Several biologic agents provided higher PASI75 rates than placebo, with golimumab yielding the most favorable results (relative risk $[R R]=14.02$ [6.85-17.11]). Accordingly, several agents provided higher ACR20 rates than placebo, with infliximab yielding the most favorable results $(R R=3.02$ [1.67-4.55]). Overall, rates of $S A E$ and $A E$ were higher for several but not all biologic agents versus placebo, with golimumab being associated with the most favorable results for $S A E$ (RR $=0.40$ $[0.11-1.41])$, and abatacept for AE (RR=1.00 [0.79-1.22]).
\end{abstract}

Conclusions: Efficacy and safety of biologic agents for psoriasis differ, and clinicians should bear in mind these features to maximize safety and efficacy in the individual patient.

Keywords: Meta-analysis, Mixed treatment comparison, Network meta-analysis, Plaque psoriasis, Psoriasis, Psoriatic arthritis, Systematic review.

\section{INTRODUCTION}

Psoriasis, whenever involving a sizable body surface of a patient or being associated with arthritis, represents a major cause of morbidity worldwide [1]. Despite the limited advancements in the management of this condition which occurred in prior decades, novel treatments have been tested in the last years, with very favorable results for many biologic agents with disease modifying properties [2]. These includes agents which block tumor necrosis factor- $\alpha$ (TNF- $\alpha$ ), as well as antilymphocyte $\mathrm{T}$, anti-interleukin-12/23 (IL-12/23), and anti-interleukin-17 (IL-17) agents. Clinicians wishing to decide which treatment is better, in terms of safety or efficacy, are however facing a major challenge, as most studies were placebo-controlled trials with moderate size, and few meaningfully powerful comparative effectiveness and safety trials are available [3].

*Address correspondence to this author at the Department of Medico-Surgical Sciences and Biotechnologies, Sapienza University of Rome, Corso della Repubblica 79, 04100 Latina, Italy; Tel: +39 07731757245;

Fax: +39 07731757254; E-mail: giuseppe.biondizoccai@uniroma1.it

E-ISSN: 1929-6029/14
Systematic reviews incorporating pairwise and network meta-analysis may successfully synthesize the evidence base on a specific clinical issue, providing precise overall and interaction effect estimates [4]. Indeed, three mixed treatment comparisons have already been reported on this topic [5-7], but were limited by the too narrow focus on a specific subset of studies, or the lack of inclusion of the many trials which have been published in the last few years. Specifically, Migliore et al. included four trials [6], Lin and colleagues 17 [5], and Reich et al. 20 [7].

We thus performed an updated and comprehensive systematic review on randomized trials focusing on biologic therapy in patients with psoriasis or psoriatic arthritis, exploiting pairwise and network meta-analytic techniques as well.

\section{METHODS}

\section{Design}

This review was conducted in compliance with the Preferred Reporting Items for Systematic Reviews and 
Meta-Analyses (PRISMA) guidelines [8]. All reviewing activities were performed independently by two experienced reviewers, with divergences solved after consensus.

\section{Search}

Pertinent studies were searched in MEDLINE/PubMed according to Biondi-Zoccai et al's string for controlled clinical trials [9], and exploiting the following terms: abatacept; adalimumab; anakinra; briakinumab; brodalumab; certolizumab; etanercept; golimumab; infliximab; ixekizumab; psoriasis; rituximab; tocilizumab; ustekinumab (see also Appendix for the detailed strategy). In addition, CENTRAL, Google Scholar, and Scopus were searched for suitable studies. The search was last updated on September 21, 2013. No language restriction was enforced.

\section{Selection}

Initially retrieved citations were screened at the title/abstract level and then retrieved as full texts if potentially pertinent. Full reports were included if reporting on patients with psoriasis receiving biologic agents, and included in a randomized trial. Studies were excluded if not based on random allocation, duplicates, lacking details on clinical efficacy or safety outcomes, including anti-IL-17 agents (whose evidence base is still preliminary and are still under preregistration investigation), or focusing on efalizumab (which was discontinued due to the risk of fatal brain infarctions associated with its usage) [10].

\section{Abstraction and Validity Appraisal}

Key baseline, procedural and outcome data were systematically retrieved, focusing specifically on efficacy and safety outcomes. As efficacy outcomes, we focused on the binary rates of reduction $\geq 75 \%$ in the Psoriasis Area and Severity Index (PASI75), and improvement $\geq 20 \%$ in the American College of Rheumatology core set of outcomes (ACR20), both at the longest available follow-up. As safety outcomes, we focused on serious adverse events (SAE), and adverse events (AE), both at the longest available follow-up. The internal validity of shortlisted studies was appraised focusing on design features, including study setting, blinding, and type of comparator.

\section{Analysis}

Categorical variables are described as counts or \%. Pairwise meta-analysis was performed with RevMan
(The Cochrane Collaboration, Copenhagen, Denmark) within a frequentist framework with the DerSimonianLaird random-effect model, pooling risk ratios $(95 \%$ confidence intervals). Conversely, network metaanalysis was performed with WinBUGS (MRC Biostatistics Unit, Cambridge, UK) within a Bayesian framework with a random-effect binomial likelihood hierarchical model, sampling effect estimates with Markov chain Monte Carlo (MCMC) methods, computing risk ratios (95\% credibility intervals) and probability of being the best treatment for each agent [11]. These analyses were based on a 50,000-run training set and a 150,000-run inferential set. Convergence was appraised with the Gelman-Rubin statistic. Model fit for Bayesian inference was appraised with the deviance information criterion (DIC), comparing random-effect and fixed-effect models reported in detail by Greco et al. [12].

Using RevMan within a frequentist framework, kairwise heterogeneity was appraised using chisquared test, and inconsistency with $\mathrm{I}^{2}$. Consistency between direct estimates (which are directly based on head-to-head randomized comparisons) and indirect estimates (which rely on the exchangeability assumption) was instead appraised by comparing consistency and inconsistency models as computed with WinBUGS in a Bayesian framework [12]. Specifically, consistency models assume that no substantial variation in treatment effect between pairwise contrasts, whereas an inconsistency model does not assume underlying similarity of direct and indirect effects. Accordingly, comparing results stemming from consistency and inconsistency models is a suitable test of the exchangeability and consistency assumptions [13]. Small study effects were appraised with funnel plot inspection using RevMan within a frequentist framework.

\section{RESULTS}

\section{Reviewing Process}

From an initial set of 21,475 citations, 21,286 were excluded at the title/abstract screening stage (Figure 1). Thereafter, 189 articles were appraised as full reports, leading to the inclusion of a total of 52 trials and 17,617 patients, including 9 different biologic agents (references of included and excluded studies are available from the corresponding author upon request). The main reason for exclusion of full reports was duplication of trial data, followed by observational design, and meta-analysis as study type. 


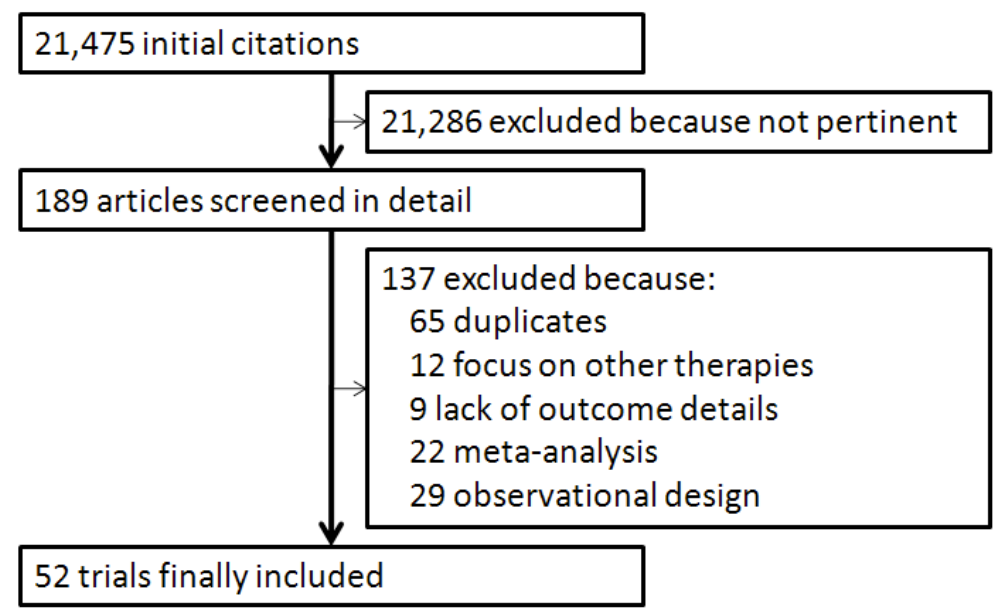

Figure 1: Review profile.

\section{Evidence Base}

The included studies compared, with variable assortments, placebo and 11 different pharmacologic agents: abatacept, acitretin, adalimumab, alefacept, briakinumab, certolizumab, etanercept, golimumab, infliximab, methotrexate, and ustekinumab (Table 1; Figure 2). Specifically, 1 trial (170 patients) compared abatacept versus placebo, 7 trials (2044) adalimumab versus placebo or control therapy, 1 (100) adalimumab versus etanercept versus infliximab, 1 (271) adalimumab versus methotrexate versus placebo, 2 (702) alafacept versus placebo, 2 (1645) briakinumab versus placebo, 2 (697) briakinumab versus etanercept versus placebo, 1 (317) briakinumab versus methotrexate, 1 (409) certolizumab pegol versus placebo, 8 (2144) etanercept versus placebo, 1 (60) etanercept versus acitretin, $1(60)$ etanercept plus acitretin versus etanercept versus acitretin, 1 (478) etanercept plus methotrexate versus etanercept alone, 1 (41) etanercept plus methotrexate versus etanercept plus cyclosporine, 1 (405) golimumab versus placebo, 9 (2006) infliximab versus placebo, 1 (868) infliximab versus methotrexate, 1 (115) infliximab plus methotrexate versus methotrexate, 7 (3358) ustekinumab versus placebo, and 1 (903) ustekinumab versus etanercept.

\section{Pairwise Meta-Analysis}

Pairwise meta-analysis for PASI75 (Figure 3) showed that adalimumab was significantly superior to placebo ( $R R=7.68$ [4.27-13.80], $\left.p<0.001, \mathrm{I}^{2}=67 \%\right)$. The same applied to alefacept $(R R=2.28 \quad$ [1.53-3.40],

Table 1: Key Biologic Agents Tested for the Treatment of Moderate to Severe Psoriasis or Psoriatic Arthritis in Randomized Clinical Trials

\begin{tabular}{|c|c|c|c|c|}
\hline Features & Agent & Manufacturer & $\begin{array}{l}\text { Route of } \\
\text { administration }\end{array}$ & Commonly used dosages and regimens in the included studies \\
\hline \multirow{2}{*}{$\begin{array}{l}\text { Anti-IL-12/23 } \\
\text { agents }\end{array}$} & Briakinumab & Abbott & SC injection & $200 \mathrm{mg}$ (or $100 \mathrm{mg}$ ) at weeks 0 and 4 followed by $100 \mathrm{mg}$ at week 8 \\
\hline & Ustekinumab & Centocor & SC injection & $90 \mathrm{mg}$ (or $45 \mathrm{mg}$ ) at week 0 , week 4 , and every 12 weeks thereafter \\
\hline \multirow[t]{2}{*}{$\begin{array}{l}\text { Anti-T-cell } \\
\text { agents }\end{array}$} & Abatacept & $\begin{array}{l}\text { Bristol Myers } \\
\text { Squibb }\end{array}$ & SC injection & $\begin{array}{c}30 / 10 \mathrm{mg} / \mathrm{kg} \text { ( } 2 \text { initial doses of } 30 \mathrm{mg} / \mathrm{kg} \text {, followed by } 10 \mathrm{mg} / \mathrm{kg} \text { ); } 10 \\
\mathrm{mg} / \mathrm{kg} \text { (or } 3 \mathrm{mg} / \mathrm{kg} \text { ) on days } 1,15 \text {, and } 29 \text { and then once every } 28 \\
\text { days }\end{array}$ \\
\hline & Alefacept & Astellas & IM injection & $15 \mathrm{mg} \mathrm{qw} ; 10 \mathrm{mg} \mathrm{qw}$ \\
\hline \multirow[t]{5}{*}{$\begin{array}{l}\text { Anti-TNF- } \alpha \\
\text { agents }\end{array}$} & Adalimumab & Abbott & SC injection & $\begin{array}{l}80 \mathrm{mg} \text { eow; } 80 \mathrm{mg} \text { loading followed by } 40 \mathrm{mg} \text { eow; } 40 \mathrm{mg} \mathrm{qw} ; 40 \\
\text { mg eow }\end{array}$ \\
\hline & $\begin{array}{l}\text { Certolizumab } \\
\text { pegol }\end{array}$ & UCB & SC injection & 400 mg qm; 400 mg eow; 200 mg eow \\
\hline & Etanercept & Amgen & SC injection & 50 mg biw; 50 mg qw; 25 mg biw; 25 mg qw; 25 mg eow \\
\hline & Golimumab & Centocor & SC injection & 100 mg qm; 50 mg qm \\
\hline & Infliximab & Centocor & IV infusion & $10 \mathrm{mg} / \mathrm{kg}$ (or $5 \mathrm{mg} / \mathrm{kg}$ or $3 \mathrm{mg} / \mathrm{kg}$ ) at weeks 0,2 , and 6 , then q6-8w \\
\hline
\end{tabular}




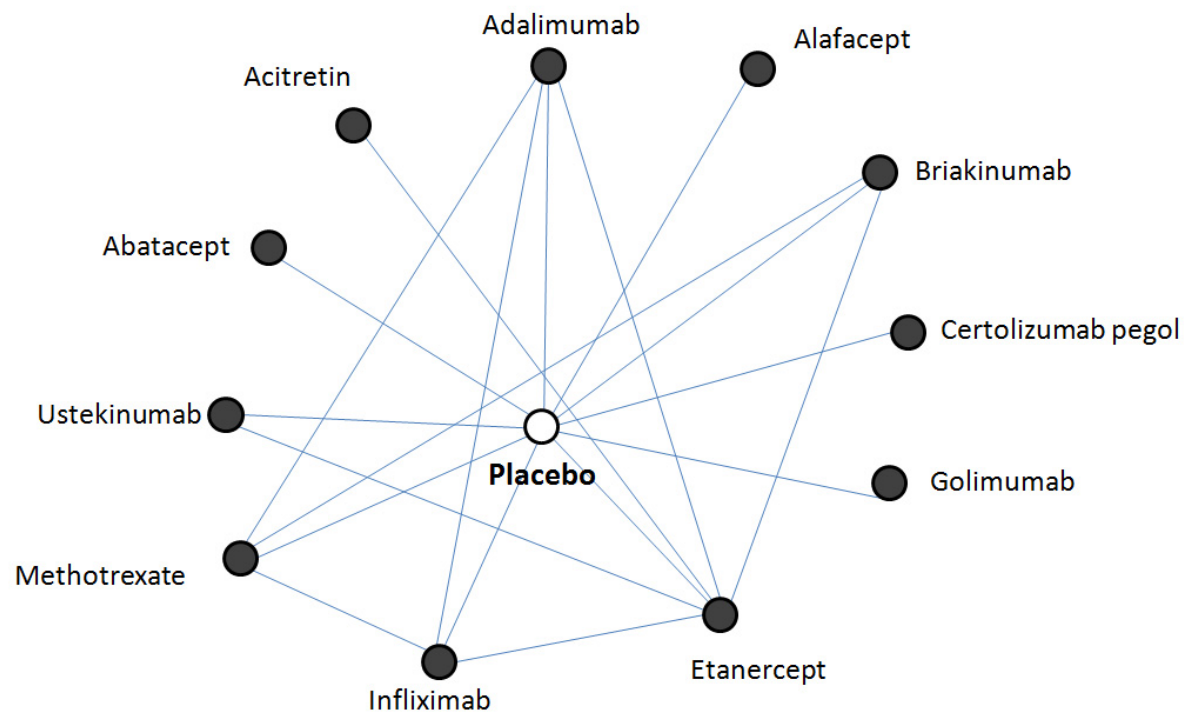

Figure 2: Evidence network.

$\left.\mathrm{p}<0.001, \mathrm{I}^{2}=0\right)$, briakinumab (RR=16.53 [11.51-23.74], $\left.p<0.001,\left.\quad\right|^{2}=0\right)$, etanercept $(R R=7.76 \quad[5.94-10.13]$, $\left.\mathrm{p}<0.001,\left.\quad\right|^{2}=0\right)$, infliximab (RR=14.52 [6.95-30.34], $\left.p<0.001, I^{2}=59 \%\right)$, and ustekinumab (RR=11.00 [6.6518.18], $\left.\quad p<0.001, \quad l^{2}=73 \%\right)$. However, funnel plot inspection suggested the presence of small study effects for PASI75 (Figure 4). Pairwise meta-analysis for ACR20 (Figure 1A) showed that adalimumab was significantly superior to placebo $(R R=3.36[2.21-5.10]$, $p<0.001, I^{2}=21 \%$ ). The same applied to etanercept $\left(R R=3.39\right.$ [2.60-6.13], $\left.p<0.001, I^{2}=0\right)$, and infliximab ( $R R=4.13$ [2.69-6.32], $p<0.001, I^{2}=4 \%$ ), without clear evidence of small study effects (Figure 2A).

Pairwise meta-analysis for SAE (Figure 3A) showed that adalimumab had a similar safety profile to placebo $\left(R R=0.92\right.$ [0.52-1.63], $\left.p=0.79, I^{2}=0\right)$. The same applied to briakinumab $\left(R R=1.21[0.57-2.57], p=0.62, I^{2}=1 \%\right)$, etanercept $\left(R R=1.21[0.57-2.56], p=0.62, l^{2}=2\right)$, and ustekinumab (RR=0.74 [0.42-1.30], $\left.p=0.29, \quad I^{2}=0\right)$. Conversely, infliximab was associated with an increased risk of $\operatorname{SAE}(R R=1.61$ [1.14-2.25], $p=0.006$, $I^{2}=0$ ). Small study effects were not apparent at funnel plot inspection (Figure 4A). Finally, pairwise metaanalysis for $A E$ showed that adalimumab had a similar safety profile to placebo $(R R=0.98$ [0.87-1.10], $p=0.75$, $I^{2}=61 \%$ ) (Figure 5A). The same applied to briakinumab $\left(R R=1.18\right.$ [0.99-1.40], $\left.p=0.06, \quad I^{2}=33 \%\right)$, etanercept $\left(R R=1.07\right.$ [0.94-1.23], $\left.p=0.31, I^{2}=0\right)$, or ustekinumab $\left(R R=1.03\right.$ [0.96-1.10], $\left.p=0.45, I^{2}=0\right)$. Conversely, AE were significantly more frequent with infliximab $\left(R R=1.17\right.$ [1.08-1.28], $\left.p<0.001, I^{2}=0\right)$. Funnel plot for $A E$ did not suggest the presence of small study effects (Figure 6A).

\section{Network Meta-Analysis}

Network meta-analysis, exploiting both direct and indirect agent-level comparisons, showed that several biologic agents provided higher PASI75 rates than placebo (Table 3 ), with golimumab yielding the most favorable results $(R R=14.02$ [6.85-17.11]). Accordingly, several agents provided higher ACR20 rates than placebo (Table 4), with infliximab yielding the most favorable results $(R R=3.02[1.67-4.55])$. Overall, rates of $S A E$ and $A E$ were higher for several but not all biologic agents versus placebo (Tables 5 and $\mathbf{6}$ ), with golimumab being associated with the most favorable results for SAE $(R R=0.40[0.11-1.41])$, and abatacept for $A E(R R=1.00[0.79-1.22])$.

\section{DISCUSSION}

This review has several key implications: first, biologic therapy for moderate to severe psoriasis or psoriatic arthritis is associated with clear and clinically meaningful benefits in terms of psoriasis and arthritis burden in comparison to placebo; second, adverse events are increased, at least by some classes of biologic agents, but the overall balance is not clearly in favor of placebo given the occurrence of diseaserelated adverse events when the condition is not adequately controlled; third, remarkable differences in safety and efficacy profile are evident between the different classes of biologic agents and even between individual agents in the same class; thus, biologic therapy should be considered in the management of moderate to severe psoriasis or psoriatic arthritis, with class and agent choice based on the specific patient risk profile, clinical history, and goal of therapy. 


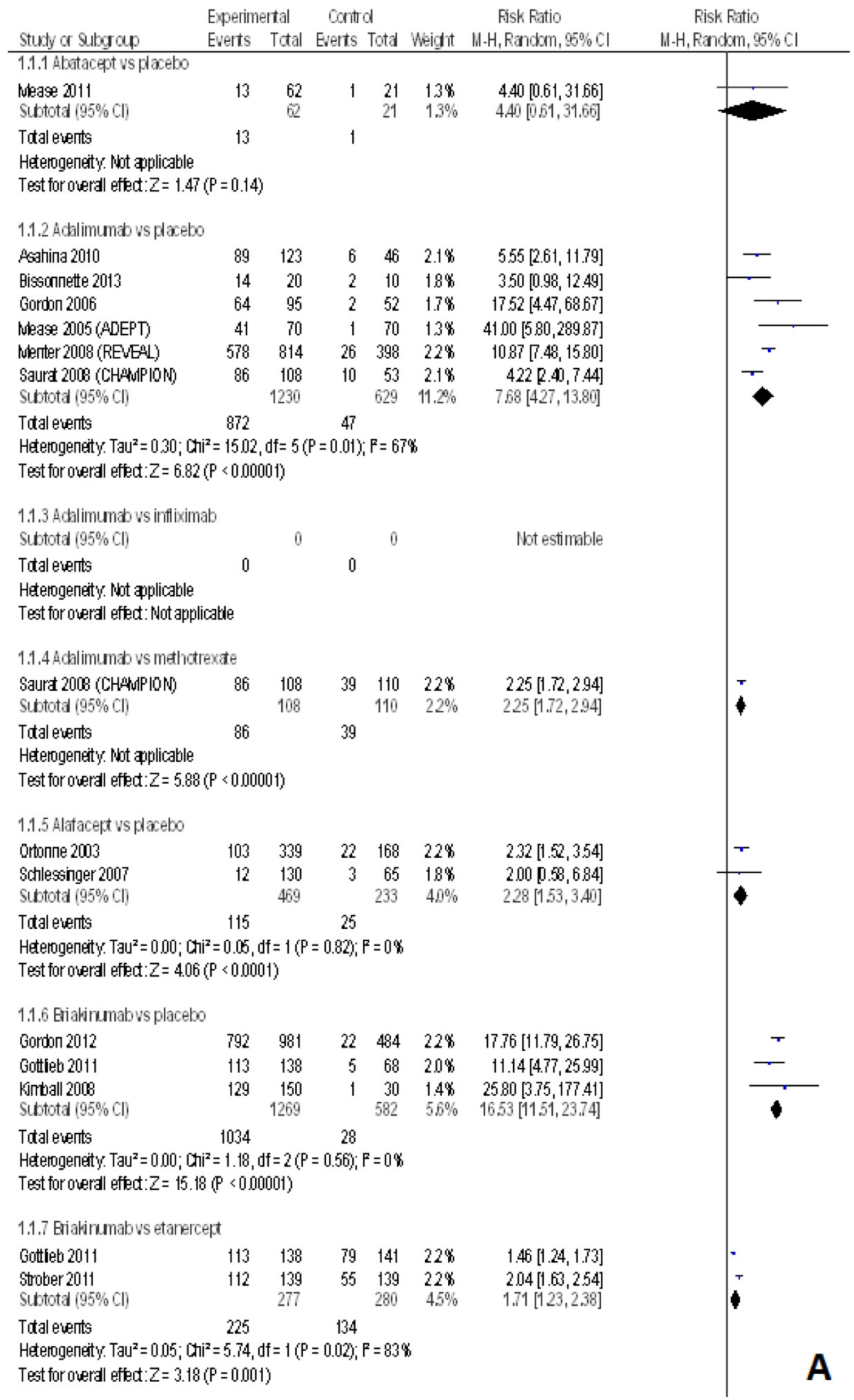


1.1.8 Eiakirumabus methotrexte

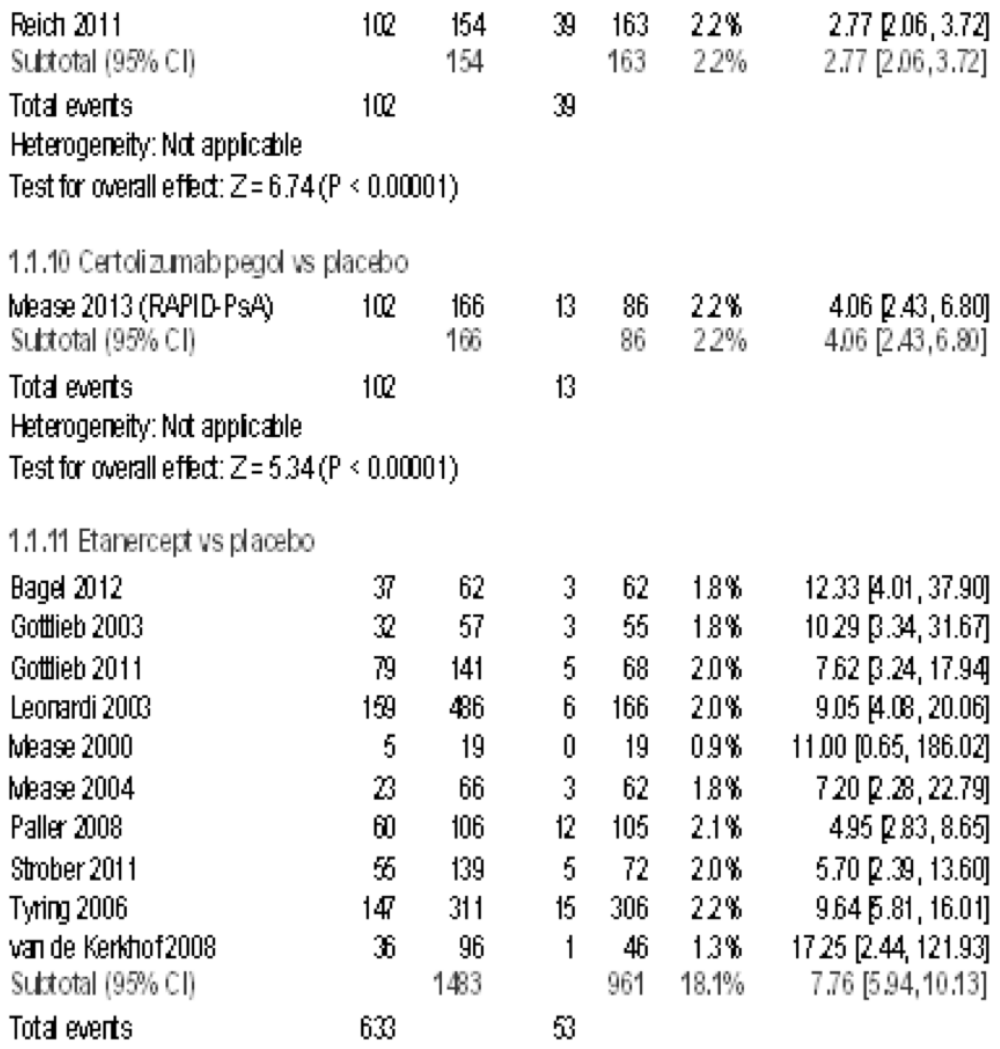

Heterogeneity: Tat $^{2}=0000 ; \mathrm{Ch}^{2}=559, \mathrm{df}=9(\mathrm{P}=0.78) \mathrm{I}^{2}=0 \%$

Test for ouerall effed: $Z=1507(P<0.00001)$

1.1.12 Etanercept vs acitretin

$\begin{array}{lrrrrrr}\text { Caprori } 2009 & 17 & 30 & 8 & 30 & 2.1 \% & 2.13[109,4.16] \\ \text { Gisond } 2008 & 8 & 18 & 6 & 20 & 20 \% & 1.48[0.54,3.45] \\ \text { Surtotal }(95 \% \mathrm{Cl}) & & 48 & & 50 & 4.1 \% & 1.85[1.99,3.13] \\ \text { Tota everts } & 5 & & 14 & & & \end{array}$

Heterogeneity: $T a u^{2}=0000 ; \mathrm{Ch}^{2}=0.43, \mathrm{df}=\left.1(\mathrm{P}=0.51)^{2}\right|^{2}=0 \%$

Test for ơuerall efted: $\mathrm{Z}=229(\mathrm{P}=0.02)$

1.1.13 Etarercept vs irfliximab

\begin{tabular}{|c|c|c|c|c|c|c|}
\hline Suntotal $(95 \% \mathrm{Cl})$ & & 0 & & \multicolumn{2}{|l|}{0} & \multirow[t]{2}{*}{ Ibt estimable } \\
\hline \multicolumn{6}{|c|}{$\begin{array}{l}\text { Heterogeneity: Not appicalo } \\
\text { Test for ouerall effed: Wot applicabl }\end{array}$} & \\
\hline \multicolumn{7}{|c|}{ 1.1.14 Etarercept us ustekirumab } \\
\hline $\begin{array}{l}\text { Griffth } 2010(A \mathrm{CCEPT}) \\
\text { Suntotal }(95 \% \mathrm{Cl})\end{array}$ & 197 & $\begin{array}{l}347 \\
34\end{array}$ & 397 & $\begin{array}{l}556 \\
556\end{array}$ & $\begin{array}{l}23 \% \\
23 \%\end{array}$ & $\begin{array}{l}0.80[0.72,0.88] \\
0.80[0.72,0.88]\end{array}$ \\
\hline Tota everts & 197 & & 397 & & & \\
\hline
\end{tabular}

Heterogeneity: Nat appicale

Test for ouverall efted: $Z=425(P<0.0001)$

1.1.15 Etanercept plus acitretin vs acitretin

$\begin{array}{llllllr}\text { Gisond } 2008 & 10 & 22 & 6 & 20 & 20 \% & 152[0.67,3.41] \\ \text { Suttotal }(95 \% \mathrm{Cl}) & & 22 & & 20 & 2.9 \% & 1.52[0.57,3.41] \\ \text { Tota euerts } & 10 & & 6 & & & \end{array}$

Heterogeneity: Nat appicale

Test for ơverall effed: $Z=100(P=0.32)$ 
(Figure 3). Continued.

1.1.16 Eareropt plus aditretin vs etanercept

$\begin{array}{lllllll}\text { Gisondi } 2008 & 10 & 22 & 8 & 18 & 2.1 \% & 1.00[051,2.04] \\ \text { Subtotal }(95 \% \mathrm{Cl}) & & 22 & & 18 & 2.1 \% & 1.02[0.51,2.04] \\ \text { Tdtal euents } & 10 & & 8 & & & \end{array}$

Heterogeneity. Not applicable

Test for oweral efed: $Z=0.06(P=0.95)$

1.1.17 Bareropt plus cyclosporine us etanercept plus methotrexte

\begin{tabular}{|c|c|c|c|c|c|c|}
\hline $\begin{array}{l}\text { Atheri } 2011 \\
\text { Subtota }(95 \% \mathrm{Cl})\end{array}$ & 10 & $\begin{array}{l}19 \\
19\end{array}$ & 7 & $\begin{array}{l}22 \\
22\end{array}$ & $\begin{array}{l}2.1 \% \\
2.1 \%\end{array}$ & $\begin{array}{l}1.65[.78,3.49 \\
1.65[0.78,3.49\end{array}$ \\
\hline \multicolumn{7}{|c|}{$\begin{array}{l}\text { Heterogeneity. Wot applicable } \\
\text { Test for oweral efed: } Z=132(P=0.19)\end{array}$} \\
\hline \multicolumn{7}{|c|}{ 1.1.18 Baneropt plus mahdrexte vs etanercept } \\
\hline $\begin{array}{l}\text { Gottlieb } 2012 \\
\text { Subtota }(95 \% \mathrm{Cl})\end{array}$ & 185 & $\begin{array}{l}239 \\
230\end{array}$ & 144 & $\begin{array}{l}239 \\
230\end{array}$ & $\begin{array}{l}23 \% \\
23 \%\end{array}$ & $\begin{array}{l}1.28[1.14,1.45 \\
1.28[1.14,1.45\end{array}$ \\
\hline Tdtal events & 185 & & 144 & & & \\
\hline
\end{tabular}

Hetergeneity. wot applicable

Test for oweral effed: $Z=397(\mathrm{P}<0.0001)$

1.1.19 Golimumab is placebo

$\begin{array}{lllllll}\text { Kawanaugh } 2009 & 127 & 208 & 1 & 73 & 13 \% & 4457 \text {. } 5.34,313.13] \\ \text { Subtota }(95 \% \mathrm{Cl}) & & 208 & & 73 & 13 \% & 44.57[6.34,313.13] \\ \text { Tdal euents } & 127 & & 1 & & & \end{array}$

Hetergeneity. Not applicable

Test for o'veral efed: $Z=382(\mathrm{P}=0.0001)$

1.1.20 Ifliximabus placebo

Antoחi 2005 (WU:PACT1)

15

Aтt0ா 2005 (')|PACT2)

$2426[1.55,378.66]$

Bisonnette 2011

$60183 \quad 1 \quad 87 \quad 13 \%$

$6289[8.9,443.47]$

Chaudari 2001

$4 \quad 12$

12

30

$4.00[052,30.76]$

Gottlieb 2004(SPIRIT)

1722

$4.25[1.19,15.19]$

$158 \quad 198 \quad 3 \quad 51 \quad 19 \%$

$13.57[452,40.75]$

puenter 2007 (EXPRES2)

$\begin{array}{lllll}457 & 627 & 4 & 208 & 19 \%\end{array}$

Beich 2005 (EXPRES1)

$227 \quad 276$

$3790[14.34,100.15]$

21.11 6.95, 64.10]

$4.89[1.70,14.02]$

Tơoii 2010

$\operatorname{rang} 2012$

Subtota $(95 \% \mathrm{Cl})$

Tdal ewents

$27 \quad 35$

$77 \quad 18 \%$

319198

$36.43[5.23,253.71]$

$14.52[6.95,30.34]$

Heterogeneity. Tau ${ }^{2}=0.70 ; 0 \mathrm{Cr}^{2}=19.63, \mathrm{df}=8(\mathrm{P}=0.01) \mathrm{I}^{2}=59 \mathrm{~s}$

Test for oweral effed: $Z=7.12(\mathrm{P}<0.00001)$

1.1.21 Ifliximabus methotrexate

$\begin{array}{lcccccc}\text { Barker 2011(REST ORED) } & 508 & 653 & 90 & 215 & 23 \% & 1.66[158,2.19] \\ \text { Subtotal }(95 \% \text { Cl) } & & 653 & & 215 & 23 \% & 186[158,2.19] \\ \text { Tdal euents } & 508 & & 90 & & & \end{array}$

Heterogeneity. Not applicable

Test for o'veral efed: $Z=7.46(\mathrm{P}<0.00001)$ 


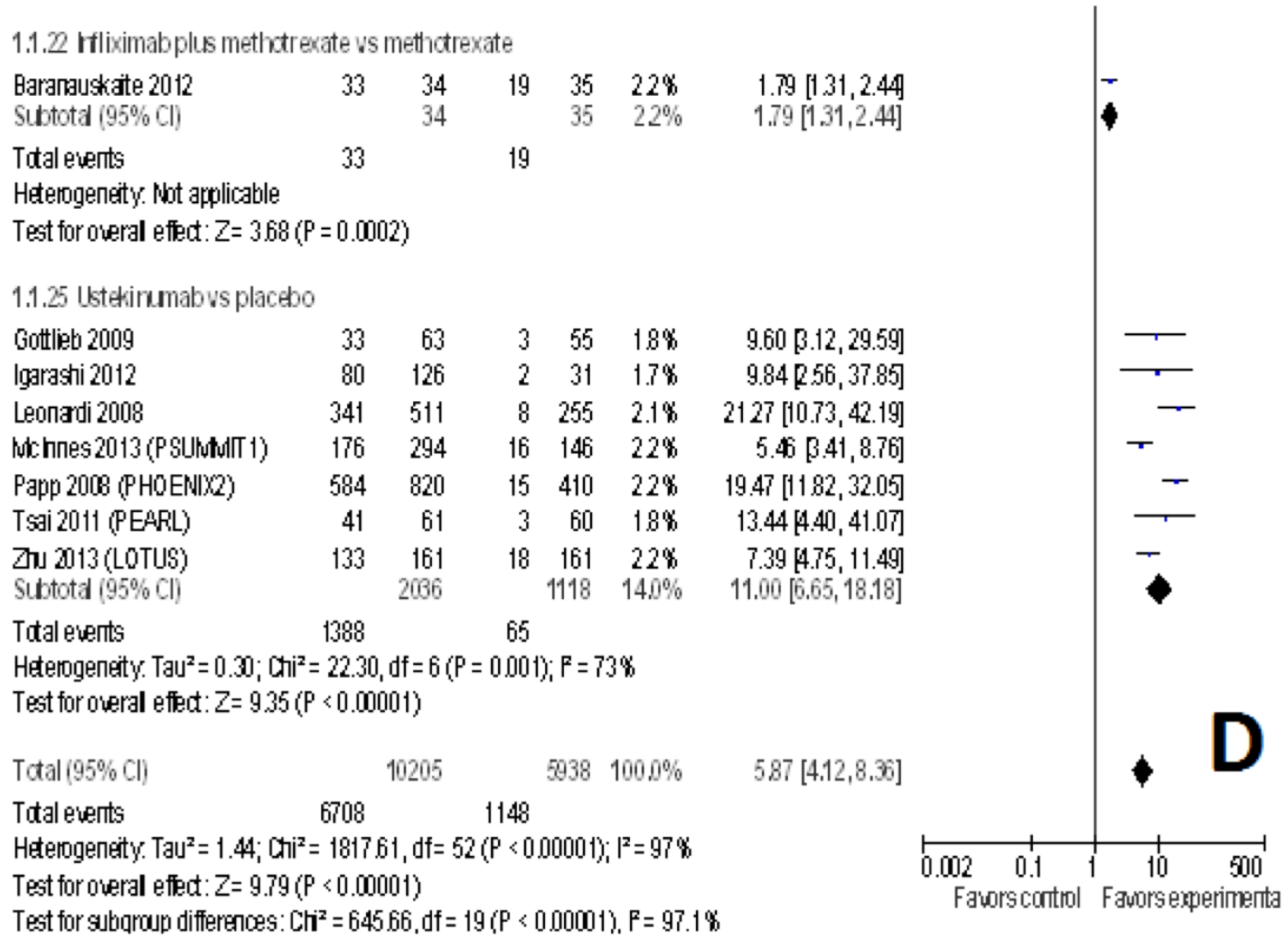

Figure 3: (panels A, B, C, and D). Forest plot for reduction $\geq 75 \%$ in the Psoriasis Area and Severity Index (PASI75). $\mathrm{Cl}=$ confidence interval; $\mathrm{df}=$ degrees of freedom; $\mathrm{M}-\mathrm{H}=$ Mantel-Haenszel.

The burden of psoriasis is very important and not limited to few developed countries. Given its chronicity and phasicity, psoriasis may prove clinically challenging, especially when associated with arthritis or involving a large part of the body surface or the nails [14]. Given the improvement in our understanding of its pathophysiology, including the preminent role of inflammation, and the setbacks of topical therapy or phototherapy in severe cases, there is an ongoing quest for effective and safe systemic therapies for psoriasis. This momentum has lead to the successful testing of several anti-inflammatory agents, and, subsequently, immune-modulating agents, typically called biologics [15].

Biologics belong to four broad categories, which correspond to the main inflammation mechanisms involved in this condition [16-19]. Agents blocking the IL-12/23 pathway, such as briakinumab and ustekinumab, anti-IL-17 agents, such as brodalumab, ixekinumab, and secukinumab, drugs which have inhibitory effects on $T$ lymphocytes, such as abatacept and alefacept, and anti-TNF- $\alpha$ agents, such as adalimumab, certolizumab pegol, etanercept, golimumab, and infliximab. Our work, which comprehensively pools the evidence on biologic agents and compare them versus placebo, acitretin, and methotrexate, has important implications for practicing physicians and patients. Under the hypothesis that each agent has, even within the same class, a unique and individual risk-benefit profile, we suggest that the most effective agent in patients with moderate to severe plaque psoriasis is golimumab, whereas the most effective one in subjects with psoriatic arthritis is infliximab. Conversely, severe adverse events were fewer with golimumab, while the occurrence of any adverse event was less likely with abatacept. However, differences between individual agents were often not large and credible. Nonetheless, decision-makers should bear in mind these agent-specific risk-benefit profiles to maximize response rates and minimize complications of systemic therapy for psoriasis.

This work is not the first in its kind, but actually builds upon prior network meta-analyses, yet substantially expanding their findings. Indeed, Lin et al. already showed, analyzing 17 trials on moderate to severe plaque psoriasis and 5 biologic agents, that ustekinumab was more efficacious than adalimumab, etanercept, and alefacept, but not infliximab [5]. 


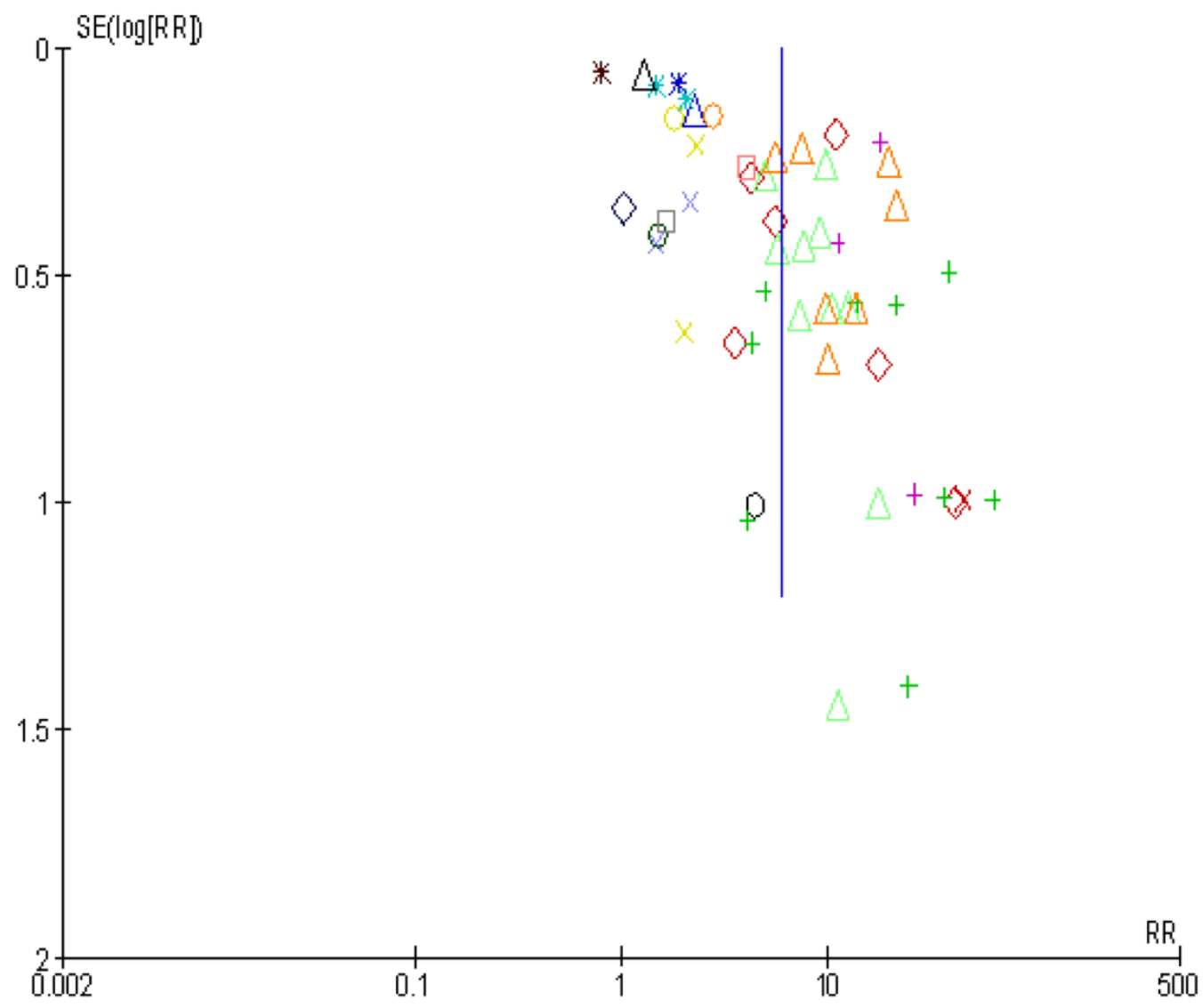

Subgroups

O Abatacept us placebo

$\bigcirc$ Adalimumab us placebo

$\square$ Addalimumab us infliximab

$\triangle$ Adalimumab us methotrexate

Alafacept us placebo

+ Briak inumab us placebo

*⿻丷木大 Briak inumab us etanercept

3 Briak inumab us methotrexate

Certolizumab pegol us placebo

Etanercept us placebo

Etanercept us acitretin

Etanercept us infliximab

* Etanercept us ustekinumab

$O$ Etanercept plus acitretin us acitretin

$\checkmark$ Etanercept plus acitretin us etanercept

$\square$ Etanercept plus cyclosporine us etanercept plus methotrexate

$\triangle$ Etanercept plus mettotrexate us etanercept

$X$ Golimumab us placebo

+ Infliximab us placebo

米 Infliximab us methotrexate

Infliximab plus methotrexate us methotrexate

Us tek inumab us placebo

Figure 4: Funnel plot for reduction $\geq 75 \%$ in the Psoriasis Area and Severity Index (PASI75). RR=relative risk; SE=standard error. 
Table 2: Key Features of Included Studies

\begin{tabular}{|c|c|c|c|c|c|}
\hline First author & Acronym & Year & Agents tested & $\begin{array}{l}\text { Sample } \\
\text { size }\end{array}$ & $\begin{array}{l}\text { Follow-up } \\
\text { (weeks) }\end{array}$ \\
\hline Antoni & IMPACT1 & 2005 & Infliximab vs placebo & 104 & 16 \\
\hline Antoni & IMPACT2 & 2005 & Infliximab vs placebo & 200 & 24 \\
\hline Asahina & & 2010 & Adalimumab vs placebo & 169 & 24 \\
\hline Atteno & & 2010 & Infliximab vs etanercept vs adalimumab & 100 & 52 \\
\hline Atzeni & & 2011 & $\begin{array}{l}\text { Etanercept plus methotrexate vs etanercept plus } \\
\text { ciclosporin }\end{array}$ & 41 & 24 \\
\hline Bagel & & 2012 & Etanarcept vs placebo & 124 & 12 \\
\hline Baranauskaite & RESPOND & 2012 & Infliximab plus methotrexate vs methotrexate & 115 & 16 \\
\hline Barker & RESTORE 1 & 2011 & Infliximab vs methotrexate & 868 & 16 \\
\hline Bissonnette & & 2011 & Infliximab vs placebo & 24 & 14 \\
\hline Bissonnette & & 2013 & Adalimumab vs control therapy & 30 & 16 \\
\hline Caproni & & 2009 & Etanercept vs acitretin & 60 & 12 \\
\hline Chaudhari & & 2001 & Infliximab vs placebo & 33 & 10 \\
\hline Genovese & & 2007 & Adalimumab vs placebo & 100 & 12 \\
\hline Gisondi & & 2008 & Etanercept vs acitretin vs etanercept plus acitretin & 60 & 24 \\
\hline Gordon & & 2006 & Adalimumab vs placebo & 148 & 12 \\
\hline Gordon & & 2012 & Briakinumab vs placebo & 1465 & 12 \\
\hline Gottlieb & & 2003 & Etanercept vs placebo & 112 & 24 \\
\hline Gottlieb & SPIRIT & 2004 & Infliximab vs placebo & 249 & 10 \\
\hline Gottlieb & & 2009 & Ustekinumab vs placebo & 146 & 12 \\
\hline Gottlieb & & 2011 & Briakinumab vs etanercept vs placebo & 347 & 12 \\
\hline Gottlieb & & 2012 & Etanercept plus methotrexate vs etanercept & 478 & 24 \\
\hline Griffiths & ACCEPT & 2010 & Ustekinumab vs etanercept & 903 & 12 \\
\hline Igarashi & & 2012 & Ustekinumab vs placebo & 158 & 12 \\
\hline Kavanaugh & GO-REVEAL & 2009 & Golimumab vs placebo & 405 & 24 \\
\hline Kimball & & 2008 & Briakinumab vs placebo & 180 & 12 \\
\hline Krueger & & 2012 & Ixekizumab vs placebo & 46 & 20 \\
\hline Leonardi & $\begin{array}{l}\text { Etanercept Psoriasis } \\
\text { Study }\end{array}$ & 2003 & Etanercept vs placebo & 672 & 12 \\
\hline Leonardi & PHOENIX1 & 2008 & Ustekinumab vs placebo & 766 & 12 \\
\hline Leonardi & $\mathrm{REACH}$ & 2011 & Adalimumab vs placebo & 72 & 16 \\
\hline Mclnnes & PSUMMIT 1 & 2013 & Ustekinumab vs placebo & 615 & 24 \\
\hline Mease & & 2000 & Etanercept vs placebo & 60 & 12 \\
\hline Mease & & 2004 & Etanercept vs placebo & 205 & 48 \\
\hline Mease & ADEPT & 2005 & Adalimumab vs placebo & 313 & 24 \\
\hline Mease & & 2011 & Abatacept vs placebo & 170 & 24 \\
\hline Mease & RAPID-PsA & 2013 & Certolizumab pegol vs placebo & 409 & 24 \\
\hline Menter & EXPRESS2 & 2007 & Inflximab vs placebo & 835 & 10 \\
\hline Menter & REVEAL & 2008 & Adalimumab vs placebo & 1212 & 16 \\
\hline Ortonne & & 2003 & Alefacept vs placebo & 507 & 14 \\
\hline Paller & & 2008 & Etanercept vs placebo & 211 & 12 \\
\hline
\end{tabular}


(Table 2). Continued.

\begin{tabular}{|c|c|c|c|c|c|}
\hline First author & Acronym & Year & Agents tested & $\begin{array}{l}\text { Sample } \\
\text { size }\end{array}$ & $\begin{array}{c}\text { Follow-up } \\
\text { (weeks) }\end{array}$ \\
\hline Papp & PHOENIX2 & 2008 & Ustekinumab vs placebo & 1230 & 12 \\
\hline Reich & EXPRESS1 & 2005 & Infliximab vs placebo & 378 & 24 \\
\hline Reich & & 2011 & Briakinumab vs methotrexate & 317 & 52 \\
\hline Saurat & CHAMPION & 2008 & Adalimumab vs methotrexate vs placebo & 271 & 16 \\
\hline Schlessinger & & 2007 & Alafacept vs placebo & 195 & 14 \\
\hline Strober & & 2011 & Briakinumab vs etanercept vs placebo & 350 & 12 \\
\hline Torii & & 2010 & Infliximab vs placebo & 54 & 14 \\
\hline Tsai & PEARL & 2011 & Ustekinumab vs placebo & 121 & 12 \\
\hline Tyring & & 2006 & Etanercept vs placebo & 618 & 12 \\
\hline van de Kerkhof & & 2008 & Etanercept vs placebo & 142 & 12 \\
\hline Yang & & 2012 & Infliximab vs placebo & 129 & 10 \\
\hline Zhu & LOTUS & 2013 & Ustekinumab vs placebo & 322 & 12 \\
\hline
\end{tabular}

Table 3: Reduction $\geq 75 \%$ in the Psoriasis Area and Severity Index (PASI75) Expressed as Decreasing Rate Ratios for Different Biologic Agents Against Placebo, and Rate Ratios Against Best Treatment, Stemming from a 5.8\% $(0.2 \%-15.2 \%)$ Rate in the Placebo Group*

\begin{tabular}{|c|c|c|}
\hline Agent & Rate ratio vs placebo & Rate ratio vs best agent (golinumab) \\
\hline \hline Golinumab & $14.02(6.85-17.11)$ & - \\
\hline Infliximab & $8.69(6.88-10.74)$ & $0.44(0.01-2.71)$ \\
\hline Briakinumab & $8.87(7.09-10.64)$ & $0.25(0.01-8.88)$ \\
\hline Ustekinumab & $7.39(5.98-8.92)$ & $0.18(0.01-1.18)$ \\
\hline Adalimumab & $6.98(5.19-8.88)$ & $0.16(0.01-1.08)$ \\
\hline Etanercept & $6.34(5.18-7.66)$ & $0.21(0.01-1.68)$ \\
\hline Abatacept & $4.99(0.93-15.77)$ & $0.10(0.01-3.51)$ \\
\hline Methotrexate & $4.55(2.98-6.37)$ & $0.09(0.01-0.60)$ \\
\hline Acitretin & $4.05(1.90-7.39)$ & $0.07(0.01-0.59)$ \\
\hline Certolizumab pegol & $3.67(1.70-7.08)$ & $0.06(0.01-0.52)$ \\
\hline Alafacept & $2.16(1.12-3.98)$ & $0.03(0.01-0.27)$ \\
\hline Placebo & - & $0.07(0.06-0.15)$ \\
\hline
\end{tabular}

${ }^{*}$ Rate ratios far from 1.0 indicate credibly different rates.

Table 4: Improvement $\mathbf{2} \mathbf{2 0} \%$ in the American College of Rheumatology Core Set of Outcomes (ACR20) Expressed as Decreasing Rate Ratios for Different Biologic Agents Against Placebo and Rate Ratios Against Best Treatment, Stemming from a $17.4 \%$ (15.1\%-19.6\%) Rate in the Placebo Group*

\begin{tabular}{|c|c|c|}
\hline Agent & Rate ratio vs placebo & Rate ratio vs best agent (infliximab) \\
\hline \hline Infliximab & $3.02(1.67-4.55)$ & $0.94(0.11-6.25)$ \\
\hline Golinumab & $2.93(0.93-4.90)$ & $0.88(0.16-4.35)$ \\
\hline Etanercept & $2.84(1.31-4.51)$ & $0.65(0.10-2.78)$ \\
\hline Adalimumab & $2.39(0.97-4.01)$ & $0.50(0.06-3.13)$ \\
\hline Certolizumab pegol & $2.03(0.56-4.22)$ & $0.44(0.05-3.13)$ \\
\hline Abatacept & $1.87(0.45-4.25)$ & $0.33(0.07-1.34)$ \\
\hline Ustekinumab & $1.41(0.56-3.09)$ & $0.33(0.22-0.60)$ \\
\hline Placebo & - & \\
\hline
\end{tabular}

${ }^{*}$ Rate ratios far from 1.0 indicate credibly different rates. 
Table 5: Serious Adverse Events (SAE) Expressed as Increasing Rate Ratios for Different Biologic Agents Against Placebo and Rate Ratios Against Best Treatment, Stemming from a $2.4 \%(1.9 \%-2.8 \%)$ Rate in the Placebo Group*

\begin{tabular}{|c|c|c|}
\hline Agent & Rate ratio vs placebo & Rate ratio vs best agent (golinumab) \\
\hline \hline Golimumab & $0.40(0.11-1.41)$ & $1.85(0.46-6.85)$ \\
\hline Ustekinumab & $0.75(0.42-1.32)$ & $2.03(0.41-8.01)$ \\
\hline Methotrexate & $0.81(0.35-1.87)$ & $2.08(0.47-8.33)$ \\
\hline Etanercept & $0.82(0.44-1.44)$ & $2.56(0.58-11.11)$ \\
\hline Adalimumab & $1.00(0.55-1.84)$ & $3.39(0.01-10.86)$ \\
\hline Briakinumab & $1.34(0.68-2.60)$ & $4.77(0.01-14.81)$ \\
\hline Infliximab & $2.00(1.16-3.52)$ & $6.23(0.01-37.51)$ \\
\hline Abatacept & $2.60(0.37-27.52)$ & $13.75(0.01-30.75)$ \\
\hline Certolizumab pegol & $6.22(2.58-14.75)$ & $2.50(0.71-9.09)$ \\
\hline Placebo & - & 0 \\
\hline
\end{tabular}

${ }^{*}$ Rate ratios far from 1.0 indicate credibly different rates.

Table 6: Adverse Events (AE) Expressed as Decreasing Rate Ratios for Different Biologic Agents Against Placebo, and Rate Ratios Against Best Treatment, Stemming from a 51.8\% (50.2\%-53.4\%) Rate in the Placebo Group*

\begin{tabular}{|c|c|c|}
\hline Agent & Rate ratio vs placebo & Rate ratio vs best agent (abatacept) \\
\hline \hline Abatacept & $1.00(0.79-1.22)$ & $1.01(0.76-1.26)$ \\
\hline Certolizumab pegol & $1.01(0.88-1.15)$ & $1.01(0.75-1.25)$ \\
\hline Ustekinumab & $1.01(0.88-1.14)$ & $1.01(0.78-1.22)$ \\
\hline Adalimumab & $1.01(0.94-1.08)$ & $1.01(0.67-1.33)$ \\
\hline Methotrexate & $1.01(0.75-1.28)$ & $1.06(0.82-1.26)$ \\
\hline Etanercept & $1.05(0.99-1.12)$ & $1.06(0.79-1.31)$ \\
\hline Golinumab & $1.06(0.92-1.21)$ & $1.06(0.84-1.28)$ \\
\hline Briakinumab & $1.06(0.99-1.13)$ & $1.09(0.86-1.30)$ \\
\hline Infliximab & $1.09(1.02-1.16)$ & $1.00(0.63-1.54)$ \\
\hline Placebo & - & \\
\hline
\end{tabular}

${ }^{*}$ Rate ratios far from 1.0 indicate credibly different rates.

Migliore and colleagues focused instead only on antiTNF-agents for psoriatic arthritis, including four trials with 820 patients. In this very specific setting, they reported that etanercept was the best agent in terms of rates of ACR20 [6]. Reich et al. pooled instead a total of 20 trials, albeit 4 of them focusing on efalizumab, which was discontinued for fatal toxicity. They suggested, in keeping with our own results, that infliximab was the drug with the most favorable efficacy profile, followed by ustekinumab, adalimumab, and etanercept [7]. Most recently, Schmitt and colleagues pooled data from 48 trials and 16,696 patients, finding that infliximab was the most effective agent for moderate-to-severe psoriasis, but limited their scope to efficacy endpoints only [20]. Finally, our findings should also be put into the cost-effectiveness context laid out in 2008 by Nelson et al., who suggested by pooling 14 trials that adalimumab and infliximab were the most cost-effective biologic agents for the treatment of psoriasis [21].

This work has several limitations, and shares most of the drawbacks typical of systematic reviews, pairwise meta-analyses, and network metaanalyses/mixed treatment comparisons [11, 12, 22]. In addition, we mainly relied on subjectively assessed endpoints, as both therapeutic response in plaque psoriasis or psoriatic arthritis is typically based on such outcomes. In addition, cross-over phases were excluded, limiting statistical precision and follow-up 
duration [23]. Notably, differences in trial phases and follow-up durations may have confounded the overall study results. Some effect estimates were based only on few studies (for instance only 1 trial reported on golimumab). Accordingly, the robustness and external validity of our results may vary depending on the specific agent analyzed and its corresponding evidence base. Appraisal of specific and rarer adverse effects of these agents (e.g. myocardial infarction, lifethreatening infection or cancer) was beyond the scope of this review [24, 25]. Finally, biologic agents can be combined with other anti-inflammatory drugs, such as methotrexate, acitretin, or cyclosporine. Other combinations include those with phototherapy or other topical treatments. Network analyses of these treatment approaches was beyond the scope of the present review and merits further investigations in the future.

In conclusion, biologic agents provide significant clinical benefits in patients with moderate to severe psoriasis or psoriatic arthritis. There are differences in the efficacy and safety profile for each agent, and clinicians should bear in mind these features to maximize safety and efficacy in the individual patient.

\section{FUNDING}

This work was supported by Novartis, Origgio, Italy.

\section{CONFLICTS OF INTEREST}

Dr. Biondi-Zoccai has consulted for Novartis, Origgio, Italy.

\section{APPENDIX}

MEDLINE/PubMed was searched according to the following explicit strategy: (psoriasis OR psoriatic) AND (abatacept OR adalimumab OR anakinra OR briakinumab OR brodalumab OR certolizumab OR etanercept OR golimumab OR infliximab OR ixekizumab OR rituximab OR tocilizumab OR ustekinumab) AND (randomized controlled trial[pt] OR controlled clinical trial[pt] OR randomized controlled trials[mh] OR random allocation[mh] OR double-blind method[mh] OR single-blind method[mh] OR clinical trial[pt] OR clinical trials[mh] OR (clinical trial[tw] OR ((singl* ${ }^{*}[\mathrm{tw}]$ OR doubl*[tw] OR trebl*[tw] OR tripl*[tw]) AND (mask* ${ }^{*}$ tw] OR blind[tw])) OR (latin square[tw]) OR placebos [mh] OR placebo*[tw] OR random*[tw] OR research design[mh:noexp] OR follow-up studies[mh] OR prospective studies[mh] OR cross-over studies[mh] OR control*[tw] OR prospectiv*[tw] OR volunteer* $\left.{ }^{*}[\mathrm{tw}]\right)$
NOT (animal[mh] NOT human[mh]) NOT (comment[pt] OR editorial[pt] OR meta-analysis[pt] OR practiceguideline[pt] OR review[pt])).

\section{SUPPLEMENTAL DATA}

The supplemental tables and figures can be downloaded from the journal website along with the article.

\section{REFERENCES}

[1] Parisi R, Symmons DP, Griffiths CE, Ashcroft DM; Identification and Management of Psoriasis and Associated ComorbidiTy (IMPACT) project team. Global epidemiology of psoriasis: a systematic review of incidence and prevalence. $J$ Invest Dermatol 2013; 133: 377-85.

http://dx.doi.org/10.1038/jid.2012.339

[2] Menter A, Gottlieb A, Feldman SR, et al. Guidelines of care for the management of psoriasis and psoriatic arthritis: Section 1. Overview of psoriasis and guidelines of care for the treatment of psoriasis with biologics. J Am Acad Dermatol 2008; 58: 826-50.

http://dx.doi.org/10.1016/j.jaad.2008.02.039

[3] Rustin $\mathrm{MH}$. Long-term safety of biologics in the treatment of moderate-to-severe plaque psoriasis: review of current data. Br J Dermatol 2012; 167: s3-11. http://dx.doi.org/10.1111/j.1365-2133.2012.11208.x

[4] Biondi-Zoccai G, Lotrionte M, Landoni G, Modena MG. The rough guide to systematic reviews and meta-analyses. HSR Proc Intensive Care Cardiovasc Anesth 2011; 3: 161-73 Available from URL: http://www.ncbi.nlm.nih.gov/pmc/ articles/PMC3484632/

[5] Lin VW, Ringold S, Devine EB. Comparison of ustekinumab with other biological agents for the treatment of moderate to severe plaque psoriasis: a bayesian network meta-analysis. Arch Dermatol 2012; 148: 1403-10.

http://dx.doi.org/10.1001/2013.jamadermatol.238

[6] Migliore A, Bizzi E, Broccoli S, Laganà B. Indirect comparison of etanercept, infliximab, and adalumimab for psoriatic arthritis: mixed treatment comparison using placebo as common comparator. Clin Rheumatol 2012; 31: 193-4. http://dx.doi.org/10.1007/s10067-011-1862-7

[7] Reich K, Burden AD, Eaton JN, Hawkins NS. Efficacy of biologics in the treatment of moderate to severe psoriasis: a network meta-analysis of randomized controlled trials. $\mathrm{Br} \mathrm{J}$ Dermatol 2012; 166: 179-88.

http://dx.doi.org/10.1111/j.1365-2133.2011.10583.x

[8] Liberati A, Altman DG, Tetzlaff J, Mulrow C, Gøtzsche PC loannidis JP, Clarke M, Devereaux PJ, Kleijnen J, Moher D. The PRISMA statement for reporting systematic reviews and meta-analyses of studies that evaluate healthcare interventions: explanation and elaboration. BMJ 2009; 339: b2700.

http://dx.doi.org/10.1136/bmj.b2700

[9] Biondi-Zoccai GG, Agostoni P, Abbate A, Testa L, Burzotta F. A simple hint to improve Robinson and Dickersin's highly sensitive PubMed search strategy for controlled clinical trials. Int J Epidemiol 2005; 34: 224-5. http://dx.doi.org/10.1093/ije/dyh311

[10] Kothary N, Diak IL, Brinker A, Bezabeh S, Avigan M, Dal Pan G. Progressive multifocal leukoencephalopathy associated with efalizumab use in psoriasis patients. J Am Acad Dermatol 2011; 65: 546-51.

http://dx.doi.org/10.1016/j.jaad.2010.05.033 
[11] D'Ascenzo F, Biondi-Zoccai G. Network meta-analyses: the "white whale" for cardiovascular specialists. J Cardiothorac Vasc Anesth 2014; 28: 169-73. http://dx.doi.org/10.1053/j.jvca.2013.01.004

[12] Greco T, Landoni G, Biondi-Zoccai G, D'Ascenzo F, Zangrillo A. A Bayesian network meta-analysis for binary outcome: how to do it. Stat Methods Med Res 2013 Oct 28 [Epub ahead of print]. http://dx.doi.org/10.1177/0962280213500185

[13] van Valkenhoef $G$, Tervonen $T$, de Brock b, Hillege $H$. Algorithmic parameterization of mixed treatment comparisons Stat Comput 2012; 22: 1099-111. http://dx.doi.org/10.1007/s11222-011-9281-9

[14] Hay RJ, Johns NE, Williams HC, et al. The global burden of skin disease in 2010: an analysis of the prevalence and impact of skin conditions. J Invest Dermatol 2013 Oct 28 [Epub ahead of print]. http://dx.doi.org/10.1038/jid.2013.446

[15] Lowes MA, Bowcock AM, Krueger JG. Pathogenesis and therapy of psoriasis. Nature 2007; 445: 866-73. http://dx.doi.org/10.1038/nature05663

[16] Schottelius AJ, Moldawer LL, Dinarello CA, Asadullah K, Sterry W, Edwards CK 3rd. Biology of tumor necrosis factoralpha- implications for psoriasis. Exp Dermatol 2004; 13: 193-222. http://dx.doi.org/10.1111/j.0906-6705.2004.00205.x

[17] Lowes MA, Kikuchi T, Fuentes-Duculan J, et al. Psoriasis vulgaris lesions contain discrete populations of Th1 and Th17 T cells. J Invest Dermatol 2008; 128: 1207-11. http://dx.doi.org/10.1038/sj.jid.5701213

[18] Nestle FO, Di Meglio P, Qin JZ, Nickoloff BJ. Skin immune sentinels in health and disease. Nat Rev Immunol 2009; 9: 679-91.

http://dx.doi.org/10.1038/nri2622

[19] Nograles KE, Zaba LC, Shemer A, et al. IL-22-producing "T22" T cells account for upregulated IL-22 in atopic dermatitis despite reduced IL-17-producing TH17 T cells. J Allergy Clin Immunol 2009; 123: 1244-52.

http://dx.doi.org/10.1016/j.jaci.2009.03.041

[20] Schmitt J, Rosumeck S, Thomaschewski G, Sporbeck B, Haufe E, Nast A. Efficacy and safety of systemic treatments for moderate-to-severe psoriasis: meta-analysis of randomized controlled trials. $\mathrm{Br} \mathrm{J}$ Dermatol 2014; 170: 274303. http://dx.doi.org/10.1111/bjd.12663

[21] Nelson AA, Pearce DJ, Fleischer AB Jr, Balkrishnan R, Feldman SR. Cost-effectiveness of biologic treatments for psoriasis based on subjective and objective efficacy measures assessed over a 12-week treatment period. J Am Acad Dermatol 2008; 58: 125-35. http://dx.doi.org/10.1016/j.jaad.2007.09.018

[22] Biondi-Zoccai G, Landoni G, Modena MG. A journey into clinical evidence: from case reports to mixed treatment comparisons. HSR Proc Intensive Care Cardiovasc Anesth 2011;3:93-6 Available from URL: http://www.ncbi.nlm.nih. gov/pmc/articles/PMC3484626/

[23] Biondi-Zoccai G. In the kingdom of the blind, the one-eyed man is king: the case for the International Journal of Statistics in Medical Research. Int J Stats Med Res 2013; 2: i-iv. Available from URL: http://www.lifescienceglobal.com/ home/cart?view $=$ product\&id $=409$

[24] Tzellos T, Kyrgidis A, Zouboulis CC. Re-evaluation of the risk for major adverse cardiovascular events in patients treated with anti-IL-12/23 biological agents for chronic plaque psoriasis: a meta-analysis of randomized controlled trials. J Eur Acad Dermatol Venereol 2013; 27: 622-7. http://dx.doi.org/10.1111/j.1468-3083.2012.04500.x

[25] Ryan C, Leonardi CL, Krueger JG, et al. Association between biologic therapies for chronic plaque psoriasis and cardiovascular events: a meta-analysis of randomized controlled trials. JAMA 2011; 306: 864-71. http://dx.doi.org/10.1001/jama.2011.1211

(C) 2014 Peruzzi et al.; Licensee Lifescience Global.

This is an open access article licensed under the terms of the Creative Commons Attribution Non-Commercial License (http://creativecommons.org/licenses/by-nc/3.0/) which permits unrestricted, non-commercial use, distribution and reproduction in any medium, provided the work is properly cited. 